\title{
Interfacing Neurons with Carbon Nanotubes: Electrical Signal Transfer and Synaptic Stimulation in Cultured Brain Circuits
}

\author{
Andrea Mazzatenta, ${ }^{1}{ }^{\star}$ Michele Giugliano, ${ }^{3 \star}$ Stephane Campidelli, ${ }^{2}$ Luca Gambazzi, ${ }^{3}$ Luca Businaro, ${ }^{4}$ Henry Markram, ${ }^{3}$ \\ Maurizio Prato, ${ }^{2}$ and Laura Ballerini ${ }^{1}$ \\ ${ }^{1}$ Physiology and Pathology Department, B.R.A.I.N., University of Trieste, I-34127, Trieste, Italy, ${ }^{2}$ Department of Pharmaceutical Sciences, University of \\ Trieste, I-34127, Trieste, Italy, ${ }^{3}$ Laboratory of Neural Microcircuitry, Brain Mind Institute, Ecole Polytechnique Fédérale de Lausanne, Station $15 \mathrm{CH}-1015$ \\ Lausanne, Switzerland, and ${ }^{4}$ LILIT BeamLine, TASC-INFM National Laboratory, Area Science Park, I-34012, Basovizza, Trieste, Italy
}

The unique properties of single-wall carbon nanotubes (SWNTs) and the application of nanotechnology to the nervous system may have a tremendous impact in the future developments of microsystems for neural prosthetics as well as immediate benefits for basic research. Despite increasing interest in neuroscience nanotechnologies, little is known about the electrical interactions between nanomaterials and neurons. We developed an integrated SWNT-neuron system to test whether electrical stimulation delivered via SWNT can induce neuronal signaling. To that aim, hippocampal cells were grown on pure SWNT substrates and patch clamped. We compared neuronal responses to voltage steps delivered either via conductive SWNT substrates or via the patch pipette. Our experimental results, supported by mathematical models to describe the electrical interactions occurring in SWNT-neuron hybrid systems, clearly indicate that SWNTs can directly stimulate brain circuit activity.

Key words: nanotechnology; hippocampal neurons; neuronal stimulation; voltage clamp; biophysical modeling; seal resistance

\section{Introduction}

Different biomedical devices implanted in the CNS, better known as neural interfaces, have been developed to control motor disorders (Benabid et al., 2005) or to translate willful brain processes into specific actions by the control of external devices (MussaIvaldi and Miller, 2003; Lebedev and Nicolelis, 2006). Clinical progresses of the numerous interface concepts proposed in basic research are limited by common recurrent problems, ranging from improper neuronal adhesion to inadequate signal stability. The success in overcoming these problems for the design of future interfaces mainly relies on the application of emerging technologies (but see also Fromherz, 2002; Stieglitz et al., 2005). Examples of current research include technologies, such as nanotechnology, that are designed to improve material/neuron junctions to be applied as implantable interfaces (Patolsky et al., 2006).

\footnotetext{
Received March 8, 2007; revised May 15, 2007; accepted May 15, 2007.

This work was supported by the Ministry of University and Research (COFIN and MUR-FIRB to M.P. and to L.B.) and EC-NEURONANO-NMP4-CT-2006-031847. We thank Dr. C. Petersen for helpful discussions, Dr. M. Hines for assistance with NEURON, Dr. L. Vaccari for assistance with scanning microscopy, Dr. S. Graziosi for computer and graphic assistance, and Drs. B. Pastore and C. Zacchigna for assistance with tissue cultures.

${ }^{*}$ A.M. and M.G. contributed equally to this work.

Correspondence should be addressed to either of the following: Dr. Laura Ballerini, Physiology and Pathology Department, Center for Neuroscience, B.R.A.I.N., University of Trieste, via Fleming 22, 34127, Trieste, Italy, E-mail: ballerin@psico.units.it; or Maurizio Prato, Department of Pharmaceutical Sciences, University of Trieste, Piazzale Europa 1, I-34127, Trieste, Italy, E-mail: prato@units.it.

D0I:10.1523/JNEUROSCI.1051-07.2007

Copyright $\odot 2007$ Society for Neuroscience $\quad$ 0270-6474/07/276931-06\$15.00/0
}

The potential of nanotechnology application in neuroscience is widely accepted (Silva, 2006). In particular, SWNTs have received great attention because of their unique physical and chemical features, which allow the development of devices with outstanding electrical properties (Krishnan et al., 1998). Recent work has focused on the feasibility of using high-capacitance, lowresistance electrodes (Gabay et al., 2007), with the goal of largescale integration with CNS interfaces (Patolsky et al., 2006). The SWNT biocompatibility has been shown previously (Mattson et al., 2000; Hu et al., 2005; Lovat et al., 2005), and neuronal adhesion, survival, and growth can be modulated by SWNT-polymer conjugates (Bekyarova et al., 2005). Nanofibers have been reported to minimize astrocyte reactions, leading to decreased glial scar tissue (McKenzie et al., 2004) in addition, the nano-scale dimension of SWNTs allows molecular interactions with neurons (Silva, 2006) that will determine the electrical interfacing of SWNTs to neural cells.

We recently demonstrated that purified carbon nanotubes are good growth surfaces for neurons and promote an increase in the efficacy of neural signal transmission (Lovat et al., 2005). Although recent reports indicate the possibility of stimulating isolated neurons in culture via SWNT (Liopo et al., 2006), the understanding of such electrical coupling is very poor. In particular, the possibility to evoke, via purified SWNT substrates, synaptic activity in long-term neural circuits is unknown.

We report here, by scanning electron microscopy, the presence of intimate contacts between cultured neurons and purified SWNT, which might provide the physical substrate for neuron-SWNT- 
neuron electrical coupling. In voltageclamped neurons, we characterize responses evoked via SWNT stimulation. Using neuronal circuits chronically grown on purified SWNT layer, we demonstrate how SWNT stimulations reliably evoke postsynaptic responses. Finally, a mathematical model incorporating SWNT and neuronal properties reproduces the main features of the response to SWNT stimulations. This model provides for the first time the basis for understanding the electrical coupling between neurons and SWNT.

\section{Materials and Methods}

Purification of SWNTs and deposition onto glass coverslips. The nanotubes (HiPCO nanotubes; Carbon Nanotechnology, Houston, TX) were functionalized via 1,3-dipolar cycloaddition reaction (Georgakilas et al., 2002), in dimethylformamide (DMF), between sarcosine, heptanal, and purified SWNT. After reaction, the nanotubes were filtered on a polytetrafluoroethylene membrane and extensively washed with DMF and dichloromethane. For deposition on the coverslips, functionalized SWNTs were resuspended in fresh DMF (using a ultrasonic bath), and few drops of the solution were deposited on the glass slides. After slow evaporation of DMF $\left(100^{\circ} \mathrm{C}\right)$, the glass coverslips were annealed at $350^{\circ} \mathrm{C}$ under $\mathrm{N}_{2}$ for $20 \mathrm{~min}$. This process permits to defunctionalize the nanotubes and fix them on the surface.

Resistivity of the SWNT deposition was estimated to be $1.0-1.2 \Omega \mathrm{mm}$, by the four-wire measurement technique and a Precision Impedance Analyzer (4294A; Agilent Technology, Santa Clara, CA). Capacitive reactance was negligible, being the phase of measured impedance $\sim 0.1^{\circ}$ in the frequency range $200-1000 \mathrm{~Hz}$. SWNT deposition with a uniform cross-section thickness of 50-60 nm was assumed, based on scanning electron microscopy investigations using focused ion beam technique (data not shown).

Tissue cultures. Standard dissociated hippocampal cultures (32 series) were prepared according to Lovat et al. (2005). Hippocampi were dissected from 0/3-d-old Sprague Dawley rats killed by decapitation. This procedure is in accordance with the relevant European Union legislation. Hippocampal slices were digested, and cells were plated on peptide-free or on SWNT-treated glass coverslips and cultured for 8-14 d (Lovat et al., 2005).

Electrophysiological recordings. Recording solution contained the following (in mM): $156 \mathrm{NaCl}, 4 \mathrm{KCl}, 1 \mathrm{MgCl}_{2}, 2 \mathrm{CaCl}_{2}, 10 \mathrm{HEPES}$, and 10 glucose (Lovat et al., 2005). Whole-cell patch-clamp recordings were obtained at room temperature using patch pipettes (4-7 M $\Omega$ ), under G $\Omega$ patch sealing, using an Axopatch 1-D (Molecular Devices, Foster City, CA). Intracellular solution contained the following (mM): 120 K-gluconate, $20 \mathrm{KCl}, 10 \mathrm{HEPES}, 10 \mathrm{EGTA}, 2 \mathrm{MgCl}_{2}$, and $2 \mathrm{Na}_{2} \mathrm{ATP}(\mathrm{pH}$ 7.35 adding $\mathrm{KOH}$ ). In voltage clamp, holding potential was set at -58 $\mathrm{mV}$. The uncompensated value for series resistance was $<8-10 \mathrm{M} \Omega$. In current-clamp recordings, bridge balancing was continuously monitored and adjusted. On a sample of 40 neurons (18 culture series), the resting membrane potential did not significantly differ between controls $(-47 \pm 2 \mathrm{mV})$ and SWNT $(-49 \pm 3 \mathrm{mV})$ neurons, as well as the input resistance $\left(R_{\mathrm{IN}}\right)$ and the cell-capacitance values $(544 \pm 50 \mathrm{M} \Omega ; 69 \pm 7 \mathrm{pF}$ and $555 \pm 54 \mathrm{M} \Omega ; 62 \pm 9 \mathrm{pF}$, glass and SWNT, respectively).

Details on electrical SWNT stimulation, electron microscopy, immunocytochemistry, and modeling are provided as supplemental Methods (available at www.jneurosci.org as supplemental material).
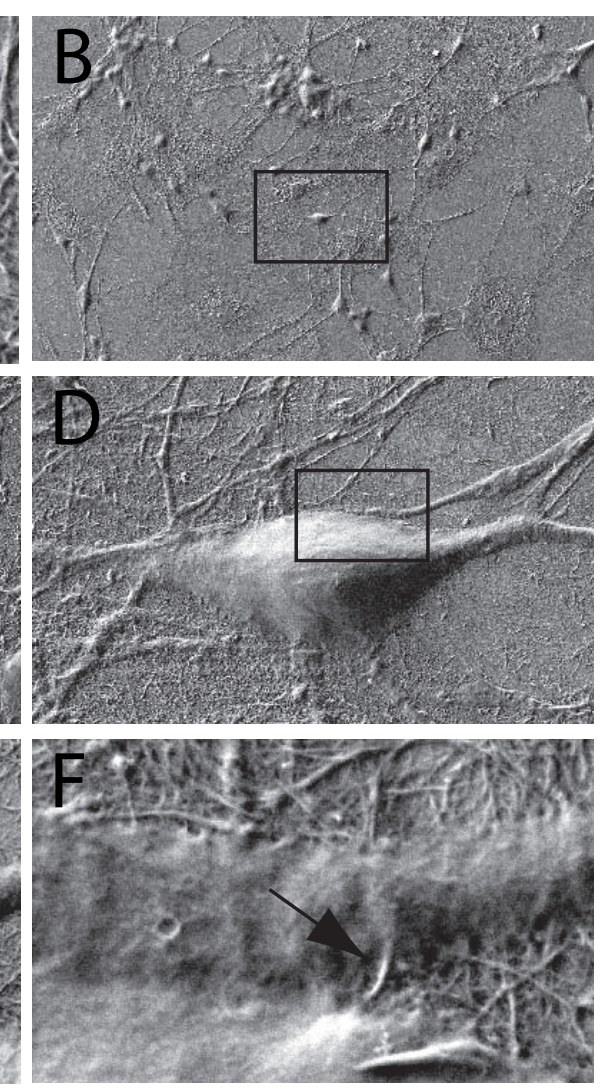

Figure 1. Scanning electron microscopy images of cultured hippocampal neurons on SWNTs. $\boldsymbol{A}$, High-magnification microas in $A$ is shown. Note the healthy morphology of neurons and the outgrowth of neurites attaching to the SWNT surface. $\boldsymbol{E}, \boldsymbol{F}$, Details of the framed area in $\boldsymbol{D}$. At higher magnifications, the intimate contacts between bundles of SWNT and neuronal membrane are clearly shown. Scale bar (in $\boldsymbol{E}$ ): $\boldsymbol{A}, 1 \mu \mathrm{m} ; \boldsymbol{B}, 200 \mu \mathrm{m} ; \boldsymbol{C}, 25 \mu \mathrm{m} ; \boldsymbol{D}, 10 \mu \mathrm{m} ; \boldsymbol{E}, 2 \mu \mathrm{m} ; \boldsymbol{F}, 450 \mathrm{~nm}$.

\section{Results}

\section{Carbon nanotubes and neuronal morphologies}

Standard conductivity measurements revealed that the SWNT layer acts as a purely resistive homogeneous network, characterized by a resistance between any two points of the order of 1-10 $\mathrm{k} \Omega$. This is consistent with scanning electron microscopy images showing that SWNTs form a dense meshwork characterized by a large surface roughness (Fig. $1 \mathrm{~A}$ ), suggesting a very large SWNTelectrolyte capacitance (Gabay et al., 2007).

We achieved direct SWNT-neuron interactions by culturing rat hippocampal cells on a film of purified SWNTs for 8-14 d, to allow for neuronal growth. Neuronal cell morphologies were analyzed by scanning electron microscopy ( $n=20$ cultures) and quantified by immunocytochemistry (see supplemental Fig. 1, available at www.jneurosci.org as supplemental material) (see also Lovat et al., 2005). In Figure $1 B-D$, scanning electron microscopy micrographs show that healthy hippocampal neurons adhered and grew on SWNT substrates displaying typical cell body morphology and size. As shown in Figure $1 B-D$, neuronal growth was accompanied by variable degree of neurite extension on the SWNT mat, similar to those observed for neurons grown on control, peptide-free glass surfaces and confirmed by immunocytochemistry analysis (supplemental Fig. 1, available at www. jneurosci.org as supplemental material) (Lovat et al., 2005). Detailed scanning electron microscopy analysis at higher magnifications of this and additional cultures suggested the presence of tight interactions between cell membranes and SWNTs at the level of neuronal processes and cell surfaces. In Fig. $1 E-F$, high- 


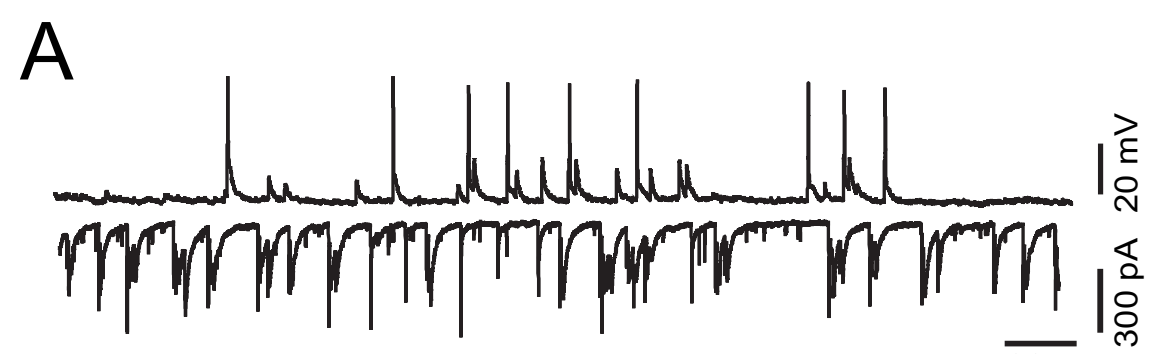

B
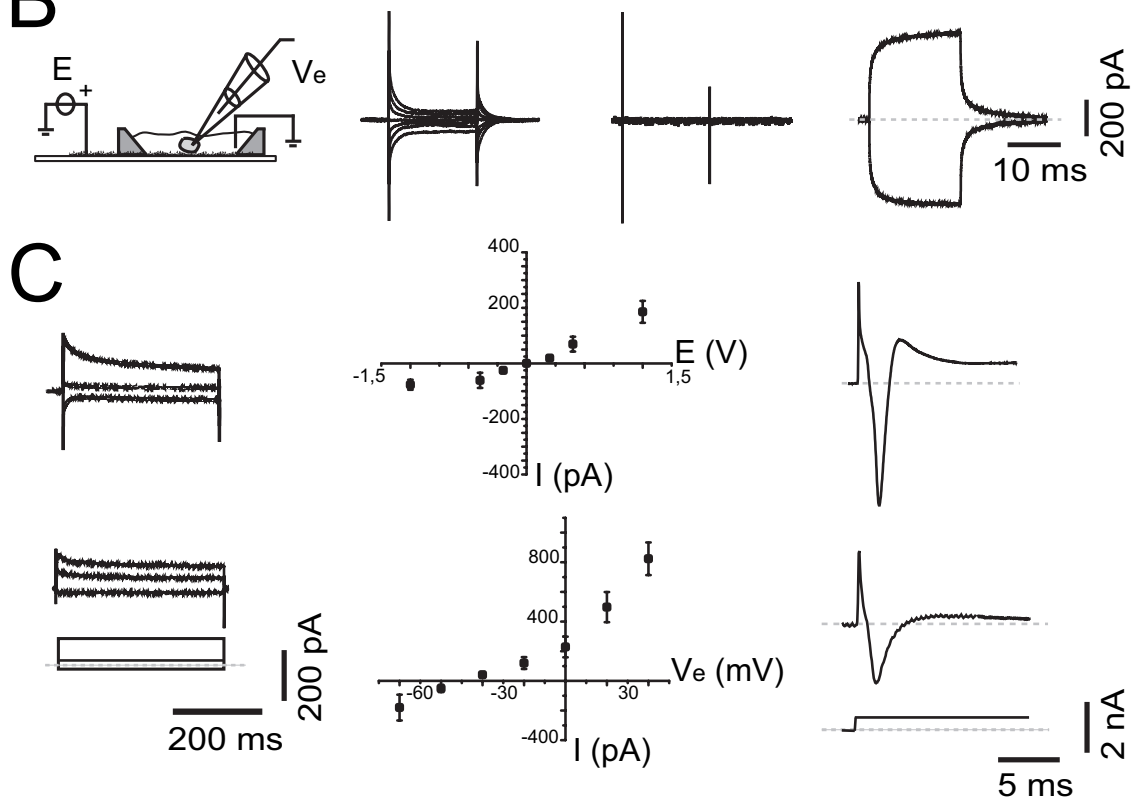

$\mathrm{D}$
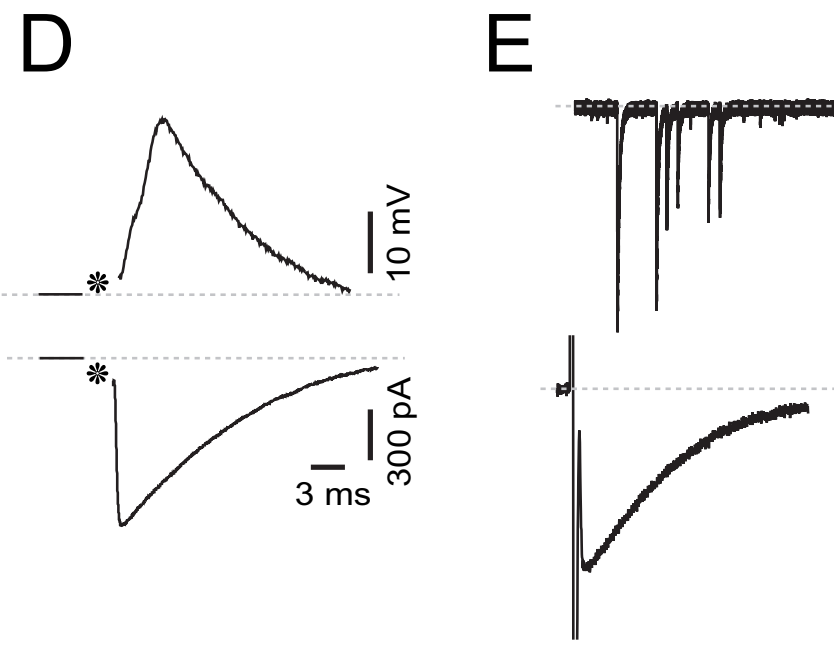

Figure 2. Electrical interactions between SWNTs and neurons. $A$, Spontaneous activity recorded from a $12 \mathrm{~d}$ cultured neuron, under current-clamp (top trace) or voltage-clamp (bottom trace) configurations. $\boldsymbol{B}$, Sketch of the recording chamber. Tracings, Current steps elicited by SWNT stimulation recorded from a patch-clamped neuron (left) or in control-glass preparation (middle) or in SWNTs before sealing to a cell (right). C, Top, Current responses to 400-ms-long SWNT stimuli in a voltage-clamped neuron, summarized in the plot ( 0.2 V bins; $n=5$ ). Bottom, Current responses in a voltage-clamped neuron elicited via the patch pipette by a step protocol, summarized in the plot (20 mV bins; $n=5)$. D, Postsynaptic events evoked via SWNT stimulation $\left(^{*}\right)$ and recorded under voltage-clamp (bottom) or current-clamp (top) configurations. $\boldsymbol{E}$, Spontaneous patterned activity and SWNT stimulation. Note that similar waveforms could be evoked via SWNT stimuli ( ${ }^{*}$ ). Bottom, Single evoked and spontaneous events, as indicated by bars.

resolution micrographs were taken at subsequent magnifications to obtain details of a region in which a presumed afferent process contacted the neuronal cell body (Fig. 1D-F). By scanning the SWNT surface topography, we observed the stable retention of
SWNT bundles (Fig. 1F, arrow). Such SWNT bundles appeared intermingled with neuronal membranes, and such a tight contact is potentially helpful in stabilizing electrical interfacing. The picture in Figure $1 F$ reports a typical example of such proximity between substrate and neuronal membranes. Although our specimens were not gold-coated to enhance EM resolution, we could resolve such structures without distortion by beam irradiation (Fig. 1F).

\section{Distributed electrical stimulation of cultured hippocampal neurons}

Hippocampal neurons, grown on SWNTs or on control glass substrates, displayed prominent spontaneous electrical activity after the first days in culture (Lovat et al., 2005). In each experiment, baseline recordings were performed from neurons displaying similar passive properties (see Materials and Methods), grown on SWNTs or on control glass substrates. Subsequently, in each SWNT neuron $(n=$ 82 ), the responses to electrical stimulation via SWNT substrates were studied by voltage-clamp and, in some cases, currentclamp recordings.

We characterized cell behavior in both glass and SWNT growth conditions. Under current clamp, a typical strong increase in the average frequency of spontaneous action potentials (APs) $(12 \pm 3 \mathrm{~Hz}$ and $44 \pm 1 \mathrm{~Hz}$, control and SWNT, respectively, $n=40$ cells, each group) was observed in neurons grown on SWNTs (Fig. $2 A$, top) (Lovat et al., 2005) when compared with controls. In Figure $2 \mathrm{~A}$ (bottom), under voltage-clamp recordings, such high firing activity is reflected by the occurrence of spontaneous PSCs, which were fully blocked by coapplication of SR-95531 (gabazine; $5 \mu \mathrm{M}$ ) and CNQX $(5 \mu \mathrm{M})$.

We did not further characterize such changes in circuit activity, because the increase in APs and frequency of PCSs are typical landmarks of neurons grown on SWNT substrates, as reported previously (Lovat et al., 2005).

To investigate neuronal responses to SWNT stimulations, we used a modified chamber with a small dry area, isolated from the recording chamber, where an $\mathrm{Ag}$ electrode was positioned in electrical contact with the SWNT film to deliver voltagecontrolled stimulation (Fig. $2 \mathrm{~B}$, sketch). In a first set of experiments, individual current responses were elicited in voltage-clamped neurons via the Ag wire-SWNT stimulation by delivering square-pulse voltage steps (20 ms duration; $0-0.3 \mathrm{~V} ; n=31$ ) (Fig. $2 B$, left) of distinct positive and negative amplitudes. As shown in Figure $2 B$ 
(middle), analogous voltage steps delivered in control-glass preparation resulted only in the appearance of fast capacitive transients on both polarities. In contrast, voltage steps delivered via SWNT induced current changes, on both polarities, when the tip of the patch electrode was simply submerged by saline solution (Fig. $2 B$, right). Thus, voltage steps applied to the SWNT layer were sensed as steady currents by the patch electrode under voltage clamp, suggesting the possible generation of redox current flows (Girault, 2004).

In Figure $2 C$ (top row, left), longer current steps with similar amplitude and polarity were obtained by delivering $400 \mathrm{~ms}$ voltage steps via SWNT to measure steadystate current values (Fig. 2C, plot, top row). Larger SWNT voltage steps induced the appearance of a fast inward current (range $1-5 \mathrm{nA}, n=12$ ) (Fig. $2 C$, top row, right), which was abolished by TTX (0.1 $\mu \mathrm{M} ; 5 \mathrm{~min})$ applications. When switching to current clamp, delivering suprathreshold SWNT stimulations induced the appearance of repetitive APs on a depolarizing step $(n=4)$ (supplemental Fig. 2, available at www.jneurosci.org as supplemental material).

Voltage steps of similar duration (from $-30 \mathrm{mV}$ to $110 \mathrm{mV}$ in $20 \mathrm{mV}$ steps; $n=$ $15)$, applied directly via the patch pipette, generated larger current responses (Fig. $2 C$, bottom, left tracings, $-10,+10,+70$ $\mathrm{mV}$ steps; see also $I / V$ plot, middle). Stronger depolarizing voltage steps delivered through the patch pipette ( $>30 \mathrm{mV}$ ) (Fig. $2 C$, bottom, right tracings) resulted again in fast inward TTX-sensitive currents (range, $1-3.5 \mathrm{nA} ; n=6)$.

Under both stimulating conditions, large inward currents probably represent unclamped APs in distal processes (Arancio et al., 1995).

The similarity among extrinsic (via SWNT) and intrinsic (via patch pipette) stimulations is intriguing and opens several questions about the nature of the coupling between SWNT and neurons; in particular, we asked whether stimulating the SWNT layer effectively generates a steady current flow in the closely attached neurons and whether the voltage-clamp configuration is the proper experimental setting to evaluate such an issue (Liopo et al., 2006).

To assess the applicability of the SWNT layer to perform local network stimulations, we delivered SWNT brief voltage steps strong enough to induce the appearance of $\mathrm{Na}^{+}$fast inward current in the recorded neuron. Such stimulations should also induce APs in neighboring neurons. Induced APs will trigger monosynaptic responses to the connected neurons, including the recorded one. In fact, short ( $2 \mathrm{~ms}$ ) SWNT voltage pulses delivered in brief trains of five consecutive steps induced, in $65 \%$ of the recorded neurons, PSCs (Fig. $2 D$, bottom) $(n=38)$ or the corresponding PSPs (top) when switching to current-clamp configuration $(n=10)$. Under both recording conditions, reversing stimulus polarity failed to evoke postsynaptic events. Evoked events were completely abolished by coapplication of SR-95531 (gabazine) and CNQX.

We then selected hippocampal networks grown on SWNTs where neurons showed the occurrence of bursts of synchronized PSCs, superimposed on a background activity, and separated by quiescent periods of variable length (Fig. $2 E$, top tracings). A brief stimulation pulse ( $2 \mathrm{~ms}$ ) delivered via the SWNT layer reliably evoked a reproducible summated PSC. Such evoked episodes displayed current waveforms similar to those of spontaneously generated ones (Fig. $2 E$, bottom tracings), suggesting the induction of similar firing patterns in the network.

\section{Modeling the neuron-SWNT junction}

Our recordings suggest that the coupling between neurons and SWNTs might be in part resistive (Liopo et al., 2006). Persistent (DC) lateral electrical stimulation delivered through the SWNT layer could (hyperpolarize) depolarize the membrane and (inhibit) elicit sustained firing in neurons intimately growing on SWNT.

To elucidate such a DC coupling, we defined, analytically studied, and numerically simulated simple resistive electrical equivalent models for the SWNT-electrolyte and the SWNTneuron junctions (Fig. 3). These models are reduced versions of detailed capacitive-resistive descriptions incorporating Hodgkin-Huxley-like currents, numerically simulated in Fig. 4. For the SWNT-electrolyte junction, we privileged a phenomenological description to a biophysical-electrochemical investigation as by Grattarola and Martinoia (1993) for metal/semicon- 


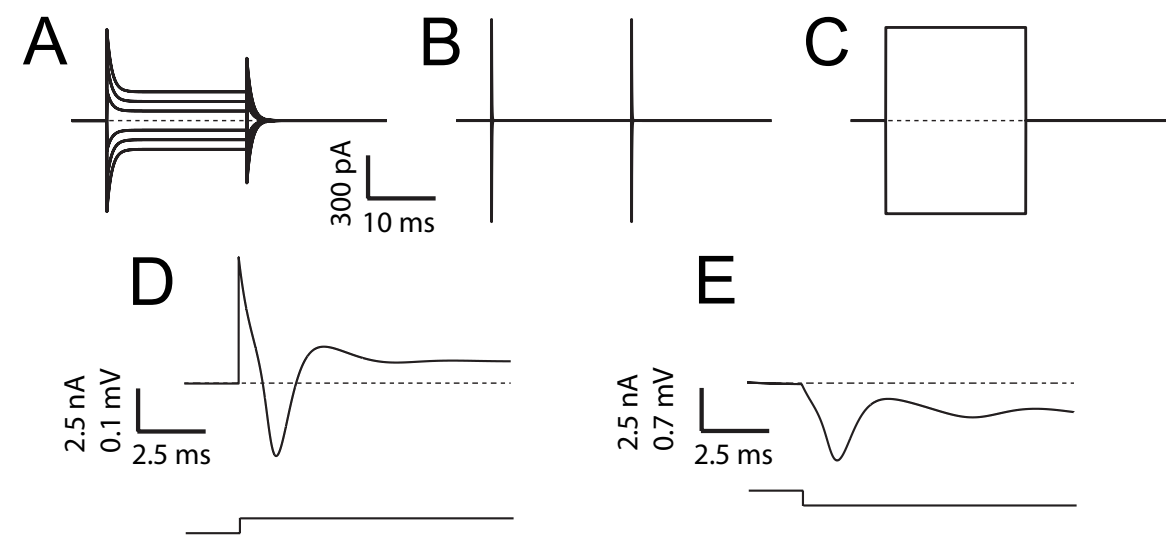

Figure 4. Simulated stimulations via the SWNT layer. Intracellular currents elicited by SWNT stimulation simulated in a model neuron in the presence $(\boldsymbol{A})$ or absence $(\boldsymbol{B})$ of SWNT and before sealing to a cell $(\boldsymbol{C})$, as in Figure 2 . The emission of single unclamped APs simulated in the models through holding voltage steps $(\boldsymbol{D})$ or by SWNT-delivered stimulation $(\boldsymbol{E})$, when incorporating the details of SEVC.

ductor-neuron interface. Nevertheless, we note that the DC properties modeled, observed, and discussed here might be attributable to oxygen chemabsorption at the SWNT-electrolyte interface (Perkins and Robinson, 2006). These reactions, not being a feature of pristine nanotubes, are probably unavoidable side effects of a variety of technological fabrication processes.

Figure 3 sketches two situations: the pipette in the bath $(A)$ or establishing a whole-cell patch $(B)$. For the first configuration, the circuit of Figure $3 C$ accounts for the DC properties of both the SWNT- and pipette-electrolyte interfaces, as confirmed experimentally (Fig. $2 B$ ). In fact, simulating single-electrode voltage clamp (SEVC), during step voltage-command, $v_{\mathrm{e}}$, through the pipette or applying a voltage step $E$ through the SWNT (Fig. 4C), one measures a current, $i_{\mathrm{e}}$, through the pipette:

$$
i_{\mathrm{e}}=v_{\mathrm{e}} /\left(R_{\mathrm{e}} / / R_{\mathrm{c}}\right)-E / R_{\mathrm{c}} .
$$

$R_{\mathrm{e}} / / R_{\mathrm{c}}$ indicates the resistances parallel of the pipette $\left(R_{\mathrm{e}} \sim 5 \mathrm{M} \Omega\right)$ and of the resistive component of the SWNT-electrolyte interface $\left(R_{\mathrm{c}} \sim 100-400 \mathrm{M} \Omega\right.$, estimated through Eq.1 and Fig. $\left.2 B\right)$, and $E$ is the voltage applied to SWNT.

In the second configuration, an ideal seal is obtained. The circuit of Figure $3 D$ models the conditions of no resistive coupling $\left(R_{\mathrm{c}}=\infty\right)$ between SWNT and the intracellular potential. Thus, $E$ has no effect on $i_{\mathrm{e}}$ as

$$
i_{\mathrm{e}}=\left(y_{\mathrm{e}}-v_{\text {rest }}\right) /\left(R_{\mathrm{e}}+R_{\mathrm{m}}\right) .
$$

$R_{\mathrm{m}}(\sim 500-600 \mathrm{M} \Omega)$ is the input resistance, and $v_{\text {rest }}$ its resting potential. This situation can be rejected on the basis of Figure $2 B$ (Liopo et al., 2006): when a step voltage $E$ is applied through SWNT, $i_{\mathrm{e}}$ indeed undergoes a steady change.

Let us now assume that a resistive pathway between the SWNT substrate and the cytoplasm exists modeled as in Figure $3 E$. Then, $E$ or $v_{\mathrm{e}}$ should induce similar perturbations (Fig. $4 A, D, E$ ). Under such hypotheses,

$$
i_{\mathrm{e}}=\left[v_{\mathrm{e}}-\left(R_{\mathrm{c}} / / R_{\mathrm{m}}\right) \cdot\left(V_{\text {rest }} / R_{\mathrm{m}}+E / R_{\mathrm{c}}\right] \cdot\left(R_{\mathrm{c}} / / R_{\mathrm{m}}+R_{\mathrm{e}}\right)^{-1} .\right.
$$

If resistive coupling occurs from a nano-scale portion of the SWNT layer, "effectively" exposed to the cytoplasm, the corresponding value of $R_{\mathrm{c}}$ is certainly much larger than those in Equation 1. If $R_{\mathrm{c}}$ is larger than the membrane resistance $R_{\mathrm{m}}$ (i.e., $\left(R_{\mathrm{c}} / /\right.$ $\left.R_{\mathrm{m}}\right) \sim R_{\mathrm{m}}$ ), Equations 2 and 3 become similar. Thus, (passive) membrane currents can be evoked by acting on $v_{\mathrm{e}}$ or by $E$, re- corded under SEVC as $i_{\mathrm{e}}$. Both $v_{\mathrm{e}}$ and $E$ appear on the right side of Equation 3, with $E$ weighted by a small factor $R_{\mathrm{m}} /$ $\left(R_{\mathrm{m}}+R_{\mathrm{c}}\right)$ (Fig. $4 A$ ). This was confirmed by the large values of $E(\sim 100-1000 \mathrm{mV})$ experimentally required to elicit currents $i_{\mathrm{e}}$ that were similar to those evoked by sweeps $10-100$ times smaller in $v_{\mathrm{e}}$ (see Fig. $2 B$ ).

What happens if we challenge the hypothesis of resistive coupling, considering the realistic case of an excellent but nonideal membrane-patch sealing? This is captured by the model circuit of Figure $3 F$, and $i_{\mathrm{e}}$ takes the following form:

$$
\begin{aligned}
i_{\mathrm{e}}=\left[v_{\mathrm{e}}\right. & -\left(R_{\mathrm{s} 2} / / R_{\mathrm{c}} / / R_{\mathrm{m}}\right) \cdot\left(v_{\text {rest }} / R_{\mathrm{m}}\right. \\
& \left.\left.\left.+E / R_{\mathrm{c}}\right)\right]_{\mathrm{s} 2} / / R_{\mathrm{c}} / / R_{\mathrm{m}}+R_{\mathrm{e}}\right)^{-1} .
\end{aligned}
$$

Interestingly, Equations 3 and 4 are identical, apart from the additional parallel resistor $R_{\mathrm{s} 2}$, which accounts for the (gigaohm-large) seal resistance of the patch pipette.

However, if we had previously assumed that no resistive coupling exists (i.e., $R_{\mathrm{c}} \sim \mathrm{M} \Omega$ ) and that the observed DC coupling occurs through the patch-seal resistance connecting the pipette tip to the extracellular SWNT, we would have derived an identical equation. In fact, although weak, the seal resistance provides a path to ground and to SWNT. In such a scenario, one could not immediately discriminate between (1) the gigaohm-large values of $R_{\mathrm{c}}$, because of the very small cytosolic domain of SWNT and (2) the series between a smaller $R_{\mathrm{c}}(\sim 100-400 \mathrm{M} \Omega)$ and a gigaohm-large seal-resistance path to the pipette tip.

Evoked PSCs in the patched cell represent the only, although indirect, evidence of SWNT induction of APs in neighboring neurons (see Fig. 2D,E). In fact, although our current-clamp recordings of sustained firing evoked by steady SWNT stimuli (supplemental Fig. 2, available at www.jneurosci.org as supplemental material) are another suggestive evidence of charge transfer between SWNT and neurons, they do not completely rule out the same caveats as for the voltage clamp. Notably, the nonidealities of series and seal resistance under SEVC can therefore hide many features of SWNT-neuron coupling (Liopo et al., 2006). Excellent but nonideal series resistance can lead to unclamped action potentials after stimulation of $E$ or $v_{\mathrm{e}}$ as experimentally observed (Figs. 2C) and replicated in the model simulations (Fig. 4). In addition, because of the intrinsic ambiguity of Equation 4, the pipette voltage can be affected equivalently by $v_{\mathrm{e}}$ and $E$, apart from appropriate scaling factors $\left(\sim R_{\mathrm{c}}\right)$.

\section{Discussion}

The main result of the present study is the new observation that neuronal circuits chronically grown on SWNT substrates can be effectively stimulated via the SWNT layers. Here, we propose for the first time a model of neuronal/SWNT coupling that should improve the understanding of bionanomaterial interactions. We specifically addressed the issue of the electrical coupling between SWNT and neurons and outlined possible deceiving results emerging from the applications of SEVC configurations in such experiments (Liopo et al., 2006). In addition, we show here the possibility to stimulate single and multiple synaptic pathways in cultured networks via SWNT platforms. Elucidating the electrical coupling between nanomaterial and neurons should also hint at 
highly targeted design of SWNT-based interfaces, minimizing unwanted interactions (Silva, 2006).

Cultured brain circuits provide an in vitro simple model of a neuronal network. Hippocampal neurons grew and developed functional circuits on SWNT surfaces, indicating, as detailed previously, the general biocompatibility of purified SWNT ( $\mathrm{Hu}$ et al., 2005; Lovat et al., 2005). When compared with control abiotic surfaces, SWNT boosted neuronal network activity under chronic growth conditions (Lovat et al., 2005). This effect has been described previously and is not attributable to differences in neuronal survival, morphology, or passive membrane properties but possibly represents a consequence of the properties of the SWNT substrate (Lovat et al., 2005). Our scanning electron microscopy observations of neuron/SWNT contacts, compared with the current state-of-the-art (Gheith et al., 2006; Liopo et al., 2006), suggested superior neuronal cell adhesion. In fact, in the absence of specific coating, the distance between electrode surfaces and cells is most likely rather large (Lu et al., 2006). However, it is not known whether our reported intimate adhesion between hippocampal neurons and SWNT affects normal cell function. Our recordings seem to exclude a long-term neurotoxicity of such interactions, because the detected spontaneous patterned activity is omnipresent in cultured neuronal circuits and has been shown to primarily result from synaptic interactions (Marom and Shahaf, 2002). This indicates that neurons, within synaptically interacting networks formed on SWNT substrates, engage in population firing. This is further strengthened by the appearance of fast $\mathrm{Na}^{+}$current, taken to constitute an early sign of axonal differentiation (Alessandri-Haber et al., 1999).

Thus far, very few investigators succeeded in growing primary neuronal cultures on SWNT substrates, none taking advantage of a purified and mechanically stable substrate, whose electrical properties could be related to SWNT properties by traditional and electrochemical methods (Gabay et al., 2007). The suggested adhesion properties between neurons and SWNTs might also sustain unconventional electrical coupling, thus unveiling new approaches to basic understanding of the CNS electrophysiology (Silva, 2006).

The electrical coupling between neurons and SWNTs is a relevant matter (Llinás et al., 2005). Our experimental results, strengthened by the modeling, supports the idea that any resistive coupling between biomembranes and SWNT (Gheith et al., 2006; Liopo et al., 2006) is qualitatively undistinguishable from a coupling between SWNT and the patch pipette through the patchseal path to ground. Because of the nonidealities of the SEVC, eliciting $\mathrm{Na}^{+}$currents through SWNT stimulation does not conclusively prove a resistive coupling between SWNT and neurons. It is indeed through detecting synaptic responses, evoked by APs elicited in not-clamped neurons, that the effectiveness of SWNTneuron interaction can be assessed. On a first approximation, regardless of the interpretative complications of the voltageclamp data, such a phenomenological observation suggests that SWNT-coated substrates might provide the best mechanical coupling between artificial devices and neural tissue.

\section{References}

Alessandri-Haber N, Paillart C, Arsac C, Gola M, Couraud F, Crest M (1999) Specific distribution of sodium channels in axons of rat embryo spinal motoneurones. J Physiol (Lond) 518:203-214.
Arancio O, Kandel ER, Hawkins RD (1995) Activity-dependent long-term enhancement of transmitter release by presynaptic $3^{\prime}, 5^{\prime}$-cyclic-GMP in cultured hippocampal neurons. Nature 376:74-80.

Bekyarova E, Ni Y, Malarkey EB, Montana V, McWilliams JL, Haddon RC, Parpura V (2005) Applications of carbon nanotubes in biotechnology biomedicine. J Biomed Nanotech 1:3-17.

Benabid AL, Wallace B, Mitrofanis J, Xia C, Piallat B, Fraix V, Batir A, Krack P, Pollak P, Berger F (2005) Therapeutic electrical stimulation of the central nervous system. C R Biol 328:177-186.

Fromherz P (2002) Electrical interfacing of nerve cells and semiconductor chips. Chemphyschem 3:276-284.

Gabay T, Ben-David M, Kalifa I, Sorkin R, Abrams ZR, Ben-Jacob E, Hanein Y (2007) Electro-chemical biological properties of carbon nanotube based multi-electrode arrays. Nanotechnology: 035201 (6 pp).

Georgakilas V, Kordatos K, Prato M, Guldi DM, Holzinger M, Hirsch A (2002) Organic functionalization of carbon nanotubes. J Am Chem Soc 124:760-761.

Gheith MK, Pappas TC, Liopo AV, Sinani V, Shim BS, Motamedi M, Wicksted, JP Kotov NA (2006) Stimulation of neural cells by lateral currents in conductive layer-by-layer films of single-walled carbon nanotubes. Adv Mater Deerfield 18:2975-2979.

Girault HH (2004) Analytical and chemical electrochemistry. New York: Marcel Dekker.

Grattarola M, Martinoia S (1993) Modeling the neuron-microtransducer junction: from extracellular to patch recording. IEEE Trans Biomed Eng 40:35-41.

Hu H, Ni Y, Mandal SK, Montana V, Zhao B, Haddon RC, Parpura V (2005) Polyethyleneimine functionalized single-walled carbon nanotubes as a substrate for neuronal growth. J Phys Chem B 109:4285-4289.

Krishnan A, Dujardin E, Ebbesen TW, Yianilos PN, Treacy MMJ (1998) Young's modulus of single-walled nanotubes. Phys Rev B 58:14013-14019.

Lebedev MA, Nicolelis MA (2006) Brain-machine interfaces: past, present future. Trends Neurosci 29:536-546.

Liopo AV, Stewart MP, Hudson J, Tour JM, Pappas TC (2006) Biocompatibility of native functionalized single-walled carbon nanotubes for neuronal interface. J Nanosci Nanotechnol 6:1365-1374.

Llinás RR, Walton KD, Nakao M, Hunter I, Anquetil PA (2005) Neurovascular central nervous recording/stimulating system: using nanotechnology probes. J Nanoparticle Res 7:111-127.

Lovat V, Pantarotto D, Lagostena L, Cacciari B, Grandolfo M, Righi M, Spalluto G, Prato M, Ballerini L (2005) Carbon nanotube substrates boost neuronal electrical signaling. Nano Lett 5:1107-1110.

Lu S, Bansal A, Soussou W, Berger TW, Madhukar A (2006) Receptorligand-based specific cell adhesion on solid surfaces: hippocampal neuronal cells on bilinker functionalized glass. Nano Lett 6:1977-1981.

Marom S, Shahaf G (2002) Development, learning memory in large random networks of cortical neurons: lessons beyond anatomy. Q Rev Biophys 35:63-87.

Mattson MP, Haddon RC, Rao AM (2000) Molecular functionalization of carbon nanotubes use as substrates for neuronal growth. J Mol Neurosci 14:175-182.

McKenzie JL, Waid MC, Shi R, Webster TJ (2004) Decreased functions of astrocytes on carbon nanofiber materials. Biomaterials 25:1309-1317.

Mussa-Ivaldi FA, Miller LE (2003) Brain-machine interfaces: computational demands clinical needs meet basic neuroscience. Trends Neurosci 26:329-334.

Patolsky F, Timko BP, Yu G, Fang Y, Greytak AB, Zheng G, Lieber CM (2006) Detection, stimulation, inhibition of neuronal signals with highdensity nanowire transistor arrays. Science 313:1100-1104.

Perkins FK, Robinson JA (2006) Chemical vapor deposition using singlewalled carbon nanotubes. Chem Soc Rev 35:790-798.

Silva GA (2006) Neuroscience nanotechnology: progress, opportunities and challenges. Nat Rev Neurosci 7:65-74.

Stieglitz T, Schuettler M, Koch KP (2005) Implantable biomedical microsystems for neural prostheses. IEEE Eng Med Biol Magn 24:58-65. 